\title{
No Existencia Global para una Ecuación de Onda Viscoelástica con Frontera Disipativa
}

\author{
Teófanes Quispe ${ }^{1}$
}

(Recibido: 19/09/2016 - Aceptado: 06/10/2016)

Resumen: Consideramos un problema mixto para una ecuación de onda no lineal viscoelástica con condiciones de frontera disipativa lineal y término fuente no lineal. Probamos la existencia y unicidad de su solución local por el método de Faedo-Galerkin y mostramos la explosión de soluciones por el método de la energía.

Palabras clave: Ecuación de onda viscoelástica, solución local, método de Faedo-Galerkin, explosión de soluciones, método de la energía.

\section{Global Nonexistence for a Viscoelastic Wave Equation with Boundary Dissipative}

Abstract: We consider a mixed problem for a viscoelastic nonilinear wave equation with boundary dissipative lineal and nonlinear source term. We prove the existence and uniqueness of its local solution by the Faedo-Galerkin method and show the blow-up of solutions by the energy method.

Keywords: Viscoelastic wave equation, local solution, Faedo-Galerkin method, blow-up of solutions, energy method.

\section{Introducción}

En este artículo, consideramos el problema para la siguiente ecuación de onda viscoelástica:

$$
\left.u^{\prime \prime}-k_{0} \Delta u+\int_{0}^{t} g(t-s) \operatorname{div}[\alpha(x) \nabla u(s)] d s+\beta(x) u^{\prime}=f(u) \quad \text { en } \Omega \times\right] 0, \infty[,
$$

con condiciones iniciales

$$
u(x, 0)=u_{0}(x), u^{\prime}(x, 0)=u_{1}(x) \text { en } \Omega,
$$

y condiciones de frontera

$$
\begin{aligned}
u(x, t) & \left.=0 \text { en } \Gamma_{0} \times\right] 0, \infty[, \\
k_{0} \frac{\partial u}{\partial \nu}-\int_{0}^{t} g(t-s)[\alpha(x) \nabla u(s)] \cdot \nu d s+\delta(x) u^{\prime} & \left.=h(u) \quad \text { en } \Gamma_{1} \times\right] 0, \infty[,
\end{aligned}
$$

donde $\Omega$ es un conjunto abierto y acotado de $\mathbb{R}^{n}$ con frontera regular $\partial \Omega=\Gamma_{0} \cup \Gamma_{1}, \Gamma_{0}$ y $\Gamma_{1}$ son cerrados, disjuntos y con medidas positivas, $\Delta$ es el operador Laplaciano, $\nabla$ es el operador gradiente, div es el operador divergencia, $g(t)$ es una función real continua no negativa para

\footnotetext{
${ }^{1}$ UNMSM, Facultad de Ciencias Matemáticas. e-mail: tquispem@unmsm.edu.pe
} 
$t \geq 0, f(s)$ y $h(s)$ son funciones reales no lineales para $s \in \mathbb{R} ; \alpha(x), \beta(x)$ y $\delta(x)$ son funciones reales positivas para $x \in \Omega, k_{0}$ es una constante positiva, $\nu$ es el vector normal unitario exterior a $\partial \Omega$. Las derivadas parciales con respecto al tiempo las denotamos: $u^{\prime}=\frac{\partial u}{\partial t}$ y $u^{\prime \prime}=\frac{\partial^{2} u}{\partial t^{2}}$.

Este problema tiene su origen en la descripción matemática de los materiales viscoelásticos. Se sabe que los materiales viscoelásticos exhiben amortiguación natural, que es debido a la propiedad especial de estos materiales para retener una memoria de su historia pasada. Desde el punto de vista matemático, estos efectos de amortiguación se modelan por los operadores íntegrodiferenciales. Por tanto, la dinámica de los materiales viscoelásticos son de gran importancia e interés ya que tienen amplias aplicaciones en las ciencias naturales. Desde el punto de vista físico, el problema $(1.1)-(1.4)$ describe la posición $u(x, t)$ de la partícula material $x$ en el tiempo $t$, que se sujeta en la porción $\Gamma_{0}$ de su frontera $\partial \Omega$ con su porción $\Gamma_{1}$ soportado por cojinetes elásticos con respuestas de frontera no lineal, representadas por la función $h(u)$ y también está sujeto a la disipación lineal representada por la función $\delta(x) u^{\prime}$. Más información al respecto, ver $[3,9]$.

Recientemente, los problemas con condiciones de frontera disipativa y/o término fuente para la ecuación de onda viscoelástica, han sido estudiados por muchos autores, y se han obtenido la existencia, comportamiento asintótico y/o explosión de soluciones. Cavalcanti et al. [2] estudiaron el problema de la forma

$$
\begin{cases}u^{\prime \prime}-\Delta u+\int_{0}^{t} g(t-s) \Delta u(s) d s=0 & \text { en } \Omega \times] 0, \infty[ \\ u(x, 0)=u_{0}(x), u^{\prime}(x, 0)=u_{1}(x) & \text { en } \Omega, \\ u(x, t)=0 & \text { en } \left.\Gamma_{0} \times\right] 0, \infty[ \\ \frac{\partial u}{\partial \nu}-\int_{0}^{t} g(t-s) \nabla u(s) \cdot \nu d s+h\left(u^{\prime}\right)=0 & \text { en } \left.\Gamma_{1} \times\right] 0, \infty[\end{cases}
$$

Ellos establecieron la existencia y la tasa de decaimiento uniforme bajo supuestos muy restrictivos en el término disipativo $h$ y la función núcleo $g$. Para condiciones más generales sobre $h$ y $g$, Cavalcanti et al. [1] demostraron la estabilidad uniforme de (1.5), siempre que $g(0)$ y $\|g\|_{L^{1}(0, \infty)}$ son lo suficientemente pequeños. Li, Zhao y Chen [5] estudiaron un problema de la forma

$$
\begin{cases}u^{\prime \prime}-\Delta u+\int_{0}^{t} g(t-s) \operatorname{div}[\alpha(x) \nabla u(s)] d s+|u|^{\gamma} u=0 & \text { en } \Omega \times] 0, \infty[ \\ u(x, 0)=u_{0}(x), u^{\prime}(x, 0)=u_{1}(x) & \text { en } \Omega, \\ u(x, t)=0 & \text { en } \left.\Gamma_{0} \times\right] 0, \infty[ \\ \frac{\partial u}{\partial \nu}-\int_{0}^{t} g(t-s)[\alpha(x) \nabla u(s)] \cdot \nu d s+h\left(u^{\prime}\right)=0 & \text { en } \left.\Gamma_{1} \times\right] 0, \infty[\end{cases}
$$

Ellos demostraron la existencia y unicidad de su solución global por el método de Galerkin y mostraron que la tasa de decaimiento uniforme de la energía. Geng y Ma [4] estudiaron el siguiente problema

$$
\begin{cases}u^{\prime \prime}-\Delta u+\int_{0}^{t} g(t-s) \operatorname{div}[\alpha(x) \nabla u(s)] d s+u^{\prime}=0 & \text { en } \Omega \times] 0, \infty[, \\ u(x, 0)=u_{0}(x), u^{\prime}(x, 0)=u_{1}(x) & \text { en } \Omega, \\ u(x, t)=0 & \text { en } \left.\Gamma_{0} \times\right] 0, \infty[, \\ \frac{\partial u}{\partial \nu}-\int_{0}^{t} g(t-s)[\alpha(x) \nabla u(s)] \cdot \nu d s=h(u) & \text { en } \left.\Gamma_{1} \times\right] 0, \infty[.\end{cases}
$$

Probaron la propiedad de explosión de soluciones, con argumentos de concavidad. Wu y Chen 
[12] estudiaron el problema de la forma

$$
\begin{cases}u^{\prime \prime}-k_{0} \Delta u+\int_{0}^{t} g(t-s) \operatorname{div}[\alpha(x) \nabla u(s)] d s+\beta(x) u^{\prime}=|u|^{p-2} u & \text { en } \Omega \times] 0, \infty[, \\ u(x, 0)=u_{0}(x), u^{\prime}(x, 0)=u_{1}(x) & \text { en } \Omega, \\ u(x, t)=0 & \text { en } \left.\Gamma_{0} \times\right] 0, \infty[, \\ k_{0} \frac{\partial u}{\partial \nu}-\int_{0}^{t} g(t-s)[\alpha(x) \nabla u(s)] \cdot \nu d s+h\left(u^{\prime}\right)=0 & \text { en } \left.\Gamma_{1} \times\right] 0, \infty[.\end{cases}
$$

Ellos establecieron la existencia global y la tasa de decaimiento general de la energía. En Tahamtani y Peyravi [10] estudiaron un problema de la forma

$$
\begin{cases}u^{\prime \prime}-k_{0} \Delta u+\int_{0}^{t} g(t-s) \operatorname{div}[\alpha(x) \nabla u(s)] d s+f\left(u^{\prime}\right)=|u|^{p-2} u & \text { en } \Omega \times] 0, \infty[, \\ u(x, 0)=u_{0}(x), u^{\prime}(x, 0)=u_{1}(x) & \text { en } \Omega, \\ u(x, t)=0 & \text { en } \left.\Gamma_{0} \times\right] 0, \infty[, \\ k_{0} \frac{\partial u}{\partial \nu}-\int_{0}^{t} g(t-s)[\alpha(x) \nabla u(s)] \cdot \nu d s+h\left(u^{\prime}\right)=0 & \text { en } \left.\Gamma_{1} \times\right] 0, \infty[,\end{cases}
$$

donde $f\left(u^{\prime}\right)=\left(k_{1}+\beta(x)\left|u^{\prime}\right|^{m-2}\right) u^{\prime}$. Con algunas restricciones sobre los datos iniciales y la función relajación, probaron que la tasa de decaimiento es similar para una cierta clase de funciones de relajación $g$. También establecieron en dos casos, la propiedad de explosión para ciertas soluciones.

Motivados por los trabajos antes mencionados, estudiamos el problema (1.1) - (1.4). Primero mostramos la existencia y la unicidad de la solución local mediante el método de Faedo-Galerkin y el método de la energía, respectivamente. Segundo probamos la propiedad de explosión de soluciones en tiempo finito, mediante el método directo de la energía, desarrollado por Li y Tsai [6], bajo algunas restricciones a los datos iniciales y para la energía inicial relativamente arbitraria. En la discusión del problema (1.1) - (1.4), empleamos las estrategias y técnicas inspiradas en los trabajos de Park and Park [7], Wu [11] y Tahamtani y Peyravi [10].

El trabajo está organizado de la siguiente manera. En la Sección 2, se establecen algunas notaciones, se mencionan resultados sin demostración y se imponen las hipótesis del trabajo. En la Sección 3, damos el teorema de existencia y unicidad de la solución local y su demostración. Finalmente en la Sección 4, enunciamos el teorema de no existencia global y su demostración.

\section{Metodología}

En esta sección presentamos algunos conceptos, notaciones, hipótesis y resultados sin demostración, los cuales serán usados en el desarrollo del presente trabajo.

Sea $\Omega$ un conjunto abierto y acotado de $\mathbb{R}^{n}$ con frontera regular $\partial \Omega$. Denotamos el producto interno en $L^{2}(\Omega)$ y $L^{2}\left(\Gamma_{1}\right)$, respectivamente por

$$
(u, v)=\int_{\Omega} u(x) v(x) d x \quad \mathrm{y} \quad(u, v)_{\Gamma_{1}}=\int_{\Gamma_{1}} u(x) v(x) d \Gamma .
$$

Denotamos por $|\cdot|_{p}$ la norma usual en $L^{p}(\Omega)$ para $1 \leq p \leq \infty$, y por $|\cdot|_{p, \Gamma_{1}}$ la norma usual en $L^{p}\left(\Gamma_{1}\right)$. Consideramos el espacio de Hilbert,

$$
H_{\Gamma_{0}}^{1}(\Omega)=\left\{v \in H^{1}(\Omega) ; v=0 \text { sobre } \Gamma_{0}\right\}
$$


provisto con el producto interno y norma dados por

$$
((u, v))=\int_{\Omega} \nabla u(x) \cdot \nabla v(x) d x \quad \text { y } \quad\|u\|=\left(\int_{\Omega}|\nabla u(x)|^{2} d x\right)^{\frac{1}{2}},
$$

respectivamente.

Sea $X$ un espacio de Banach, $T$ y $p$ números reales tales que $0<T \leq \infty$ y $1 \leq p \leq \infty$. Representamos con $L^{p}(0, T ; X)$ al espacio de Banach de las funciones vectoriales $\left.u:\right] 0, T[\longrightarrow$ $X$ medibles con $\|u(t)\|_{X} \in L^{p}(0, T)$, dotado de la norma

$$
\begin{gathered}
\|u\|_{L^{p}(0, T ; X)}=\left(\int_{0}^{T}\|u(t)\|_{X}^{p} d t\right)^{\frac{1}{p}}, 1 \leq p<\infty, \\
\|u\|_{L^{\infty}(0, T ; X)}=\sup _{0<t<T} \operatorname{ess}\|u(t)\|_{X}, p=\infty .
\end{gathered}
$$

Similarmente, cuando $0<T<\infty$, representamos con $C([0, T] ; X)$ al espacio de Banach de las funciones continuas $u:[0, T] \longrightarrow X$, provisto de la norma

$$
\|u\|_{C([0, T] ; X)}=\sup _{0 \leq t \leq T}\|u(t)\|_{X} .
$$

Denotamos las derivadas parciales con respecto al tiempo: $w^{\prime}=\frac{\partial w}{\partial t} \mathrm{y} w^{\prime \prime}=\frac{\partial^{2} w}{\partial t^{2}}, \mathrm{y}$ escribimos $w(t)(x)=w(x, t)$.

Hipótesis. Imponemos sobre las funciones $\alpha, \beta, \delta, g, f$ y $h$ las siguientes condiciones:

$\left(H_{1}\right) \alpha, \beta: \Omega \rightarrow \mathbb{R}$ y $\delta: \Gamma_{1} \rightarrow \mathbb{R}$ son funciones positivas y $\alpha \in C^{1}(\bar{\Omega}), \beta \in L^{\infty}(\Omega), \delta \in L^{\infty}\left(\Gamma_{1}\right)$ tales que

$$
0<\alpha_{0} \leq \alpha(x) \text { y } 0<\delta_{0} \leq \delta(x) .
$$

$\left(H_{2}\right) g:[0, \infty) \rightarrow[0, \infty)$ es una función no creciente de clase $C^{2}$ tal que

$$
g(0)>0, \quad k_{0}-\|\alpha\|_{L^{\infty}(\Omega)} \int_{0}^{\infty} g(s) d s=l>0
$$

y existen constantes positivas $\xi_{1}, \xi_{2}$ y $\xi_{3}$ tales que

$$
-\xi_{1} g(t) \leq g^{\prime}(t) \leq-\xi_{2} g(t), \forall t \geq 0
$$

y

$$
0 \leq g^{\prime \prime}(t) \leq \xi_{3} g(t), \forall t \geq 0 .
$$

$\left(H_{3}\right) f, h: \mathbb{R} \rightarrow \mathbb{R}$ son funciones de clase $C^{1}$ tales que $f(0)=0, h(0)=0$ y existen constantes positivas $N_{0}$ y $M_{0}$ tales que

$$
\begin{aligned}
& |f(x)-f(y)| \leq N_{0}\left(|x|^{p}+|y|^{p}\right)|x-y|, \forall x, y \in \mathbb{R}, \\
& |h(x)-h(y)| \leq M_{0}\left(|x|^{q}+|y|^{q}\right)|x-y|, \forall x, y \in \mathbb{R},
\end{aligned}
$$

donde

$$
\begin{aligned}
& 0<p \leq \frac{2}{n-2} \text { para } n \geq 3 \text { y } p>0 \text { para } n=1,2, \\
& 0<q \leq \frac{1}{n-2} \text { para } n \geq 3 \text { y } q>0 \text { para } n=1,2 .
\end{aligned}
$$


$\left(H_{4}\right)$ Existe una constante positiva $\gamma \geq \frac{k_{0}-l}{4 l}$ tal que

$$
s f(s) \geq 2(2 \gamma+1) F(s) \quad \text { y } \operatorname{sh}(s) \geq 2(2 \gamma+1) H(s), \forall s \in \mathbb{R},
$$

donde

$$
F(s)=\int_{0}^{s} f(\xi) d \xi \quad \text { y } H(s)=\int_{0}^{s} h(\xi) d \xi
$$

Lema 2.1 (Desigualdad de Sobolev-Poincaré). Si $1 \leq q \leq \frac{2 n}{n-2}$ para $n \geq 3$ y $1 \leq q<\infty$ para $n \leq 2$, entonces existe una constante positiva $B_{1}$ tal que

$$
|u|_{q} \leq B_{1}\|u\|, \forall u \in H_{\Gamma_{0}}^{1}(\Omega) .
$$

Si $1 \leq q \leq \frac{2(n-1)}{n-2}$ para $n \geq 3$ y $1 \leq q<\infty$ para $n \leq 2$, entonces existe una constante positiva $B_{2}$ tal que

$$
|u|_{q, \Gamma_{1}} \leq B_{2}\|u\|, \forall u \in H_{\Gamma_{0}}^{1}(\Omega) .
$$

Lema 2.2 (Desigualdad generalizada de Gronwall). Sea $f:[0, \infty[\rightarrow[0, \infty[$ continua, $g:] 0, \infty[\rightarrow] 0, \infty[$ continua y no decreciente y sea $C$ una constante positiva. Si

$$
f(t) \leq C+\int_{0}^{t} g(f(s)) d s, 0 \leq t<\infty,
$$

entonces

$$
f(t) \leq G^{-1}\left(T_{*}\right)<\infty, 0 \leq t \leq T_{*},
$$

para cualquier número fijo $T_{*}<G(\infty)$, donde

$$
G(\tau)=\int_{C}^{\tau} \frac{1}{g(s)} d s, \text { para } \tau \geq C .
$$

Además, si $G(\infty)=\infty$, entonces

$$
f(t) \leq G^{-1}(t), \text { para todo } t \geq 0 .
$$

Lema 2.3 (Teorema de la divergencia). Sea $\Omega$ un conjunto abierto y acotado de $\mathbb{R}^{n}$ con frontera regular $\partial \Omega$. Si $U \in\left(H^{1}(\Omega)\right)^{n} y w \in H^{1}(\Omega)$, entonces

$$
\int_{\Omega} w(x) \operatorname{div} U(x) d x=\int_{\partial \Omega} w(x) U(x) \cdot \eta d \Gamma-\int_{\Omega} U(x) \cdot \nabla w(x) d x,
$$

donde $\eta$ es el vector normal unitaria exterior a $\partial \Omega$.

Lema 2.4. Si $g \in C^{1}\left(\left[0, \infty[)\right.\right.$ y $w \in C^{1}\left([0, T] ; L^{2}(\Omega)\right)$, entonces

$$
\begin{aligned}
\int_{0}^{t} g(t-s)\left(\alpha w(s), w^{\prime}(t)\right) d s= & -\frac{1}{2} \frac{d}{d t}\left[(g \square w)(t)-\left(\int_{0}^{t} g(s) d s\right)|\sqrt{\alpha} w(t)|_{2}^{2}\right] \\
& -\frac{1}{2} g(t)|\sqrt{\alpha} w(t)|_{2}^{2}+\frac{1}{2}\left(g^{\prime} \square w\right)(t),
\end{aligned}
$$

donde

$$
(v \square w)(t)=\int_{0}^{t} v(t-s)|\sqrt{\alpha}(w(t)-w(s))|_{2}^{2} d s .
$$


Lema 2.5 ([6]). Sea $\gamma>0$ y $B \in C^{2}([0, \infty[)$ una función no negativa que satisface

$$
B^{\prime \prime}(t)-4(\gamma+1) B^{\prime}(t)+4(\gamma+1) B(t) \geq 0, \text { para } t \geq 0 .
$$

Si $B^{\prime}(0)>r_{2} B(0)+K_{0}$, entonces $B^{\prime}(t)>K_{0}$, para $t>0$, donde $K_{0}$ es una constante $y$

$$
r_{2}=2(\gamma+1)-2 \sqrt{(\gamma+1) \gamma}
$$

es la menor raíz de la ecuación cuadrática $r^{2}-4(\gamma+1) r+4(\gamma+1)=0$.

Lema 2.6 $([6])$. Si $J(t)$ es una función no creciente en $\left[t_{0}, \infty\left[, t_{0} \geq 0\right.\right.$ y satisface la inecuación diferencial

$$
\left[J^{\prime}(t)\right]^{2} \geq a+b[J(t)]^{2+\frac{1}{\gamma}}, \text { para } t \geq t_{0},
$$

donde $a>0, \gamma>0$ y $b \in \mathbb{R}$, entonces existe un número real positivo $T_{*}$ tal que

$$
\lim _{t \rightarrow T_{*}^{-}} J(t)=0
$$

y una cota superior de $T_{*}$ puede ser estimada respectivamente, en los siguientes casos:

(i) Si $b<0$ y $J\left(t_{0}\right)<\operatorname{mín}\left\{1, \sqrt{\frac{a}{-b}}\right\}$, entonces

$$
T_{*} \leq t_{0}+\frac{1}{\sqrt{-b}} \ln \left(\frac{\sqrt{\frac{a}{-b}}}{\sqrt{\frac{a}{-b}}-J\left(t_{0}\right)}\right) .
$$

(ii) Si $b=0$, entonces

$$
T_{*} \leq t_{0}+\frac{J\left(t_{0}\right)}{\sqrt{a}} .
$$

(iii) Si $b>0$, entonces

$$
T_{*} \leq \frac{J\left(t_{0}\right)}{\sqrt{a}}
$$

o

$$
T_{*} \leq t_{0}+2^{\frac{3 \gamma+1}{2 \gamma}} \frac{\gamma c}{\sqrt{a}}\left(1-\left[1+c J\left(t_{0}\right)\right]^{-\frac{1}{2 \gamma}}\right)
$$

donde $c=\left(\frac{a}{b}\right)^{2+\frac{1}{\gamma}}$

\section{Resultados y Discusión}

\section{Existencia Local}

En esta sección, discutiremos la existencia y la unicidad de la solución local del problema (1.1) (1.4), usando el método de Faedo-Galerkin.

Teorema 3.1 (Existencia local). Supongamos que se verifican las hipótesis $\left(H_{1}\right)-\left(H_{3}\right)$ y $u_{0} \in H_{\Gamma_{0}}^{1}(\Omega) \cap H^{2}(\Omega), u_{1} \in H_{\Gamma_{0}}^{1}(\Omega)$ satisfacen la condición de compatibilidad

$$
\frac{\partial u_{0}}{\partial \nu}+\delta(x) u_{1}=h\left(u_{0}\right) \text { sobre } \Gamma_{1} .
$$


Entonces el problema (1.1) - (1.4) tiene una única solución fuerte u en la clase

$$
\begin{aligned}
& u \in L^{\infty}\left(0, T_{0} ; H_{\Gamma_{0}}^{1}(\Omega) \cap H^{2}(\Omega)\right), \\
& u^{\prime} \in L^{\infty}\left(0, T_{0} ; H_{\Gamma_{0}}^{1}(\Omega)\right), \\
& u^{\prime \prime} \in L^{\infty}\left(0, T_{0} ; L^{2}(\Omega)\right) .
\end{aligned}
$$

Además, si $u_{0} \in H_{\Gamma_{0}}^{1}(\Omega)$ y $u_{1} \in L^{2}(\Omega)$, entonces el problema $(1.1)$ - (1.4) tiene una única solución débil u en la clase

$$
u \in C\left(\left[0, T_{0}\right) ; H_{\Gamma_{0}}^{1}(\Omega)\right) \cap C^{1}\left(\left[0, T_{0}\right) ; L^{2}(\Omega)\right),
$$

para algún $T_{0}>0$.

Demostración. Abordamos la demostración en cinco etapas, como sigue:

Soluciones aproximadas. Sea $\left(w_{k}\right)_{k \in \mathbb{N}}$ una base en $H_{\Gamma_{0}}^{1}(\Omega) \cap H^{2}(\Omega)$ que es ortonormal en $L^{2}(\Omega)$ y $V_{m}$ el subespacio de $H_{\Gamma_{0}}^{1}(\Omega) \cap H^{2}(\Omega)$ generado por los primeros $m$ vectores $w_{1}, w_{2}, \ldots, w_{m}$ de $\left(w_{k}\right)_{k \in \mathbb{N}}$. Sea

$$
u_{m}(t)=\sum_{j=1}^{m} r_{j m}(t) w_{j}
$$

las soluciones aproximadas en $V_{m}$ del problema (1.1) - (1.4), donde las funciones $r_{j m}(t), j=$ $1,2, \ldots, m$, son determinadas del siguiente problema en ecuaciones diferenciales ordinarias, para $w \in V_{m}$

$$
\left\{\begin{aligned}
\left(u_{m}^{\prime \prime}(t), w\right)+k_{0}\left(\left(u_{m}(t), w\right)\right)+ & \left(\beta u_{m}^{\prime}, w\right)-\int_{0}^{t} g(t-s)\left(\alpha \nabla u_{m}(s), \nabla w\right) d s \\
& +\left(\delta u_{m}^{\prime}, w\right)_{\Gamma_{1}}=\left(f\left(u_{m}\right), w\right)+\left(h\left(u_{m}\right), w\right)_{\Gamma_{1}}, \\
u_{m}(0)=u_{0 m}, u_{m}^{\prime}(0)=u_{1 m}, &
\end{aligned}\right.
$$

donde

$$
\begin{aligned}
& u_{0 m}=\sum_{j=1}^{m} r_{0 j m} w_{j}, \quad u_{0 m} \rightarrow u_{0} \quad \text { fuerte en } H_{\Gamma_{0}}^{1}(\Omega) \cap H^{2}(\Omega), \\
& u_{1 m}=\sum_{j=1}^{m} r_{1 j m} w_{j}, \quad u_{1 m} \rightarrow u_{1} \quad \text { fuerte en } H_{\Gamma_{0}}^{1}(\Omega) .
\end{aligned}
$$

Por el Teorema de Carathéodory, aseguramos la existencia de una solución local $u_{m}$ del problema aproximado (3.2) en algún intervalo $\left[0, T_{m}\left[\right.\right.$. Además se tiene que $r_{j m}(t)$ y $r_{j m}^{\prime}(t)$ son absolutamente continuas y $r_{j m}^{\prime \prime}(t)$ existe c.t.p. en $\left[0, T_{m}[\right.$. Las siguientes estimativas a priori nos permitirá extender la solución $u_{m}$ a un intervalo $\left[0, T_{0}\right]$ independiente de $m$.

Primera estimativa. Tomando $w=u_{m}^{\prime}(t)$ en (3.2), obtenemos

$$
\begin{aligned}
\frac{1}{2} \frac{d}{d t}\left|u_{m}^{\prime}(t)\right|_{2}^{2}+ & \frac{k_{0}}{2} \frac{d}{d t}\left\|u_{m}(t)\right\|_{2}^{2}+\left|\sqrt{\beta} u_{m}^{\prime}(t)\right|_{2}^{2} \\
-\int_{0}^{t} g(t-s) & \left(\alpha \nabla u_{m}(s), \nabla u_{m}^{\prime}(t)\right) d s+\left|\sqrt{\delta} u_{m}^{\prime}(t)\right|_{2, \Gamma_{1}}^{2} \\
& =\left(f\left(u_{m}\right), u_{m}^{\prime}(t)\right)+\left(h\left(u_{m}\right), u_{m}^{\prime}(t)\right)_{\Gamma_{1}} .
\end{aligned}
$$


Del Lema 2.4, resulta

$$
\begin{aligned}
-\int_{0}^{t} g(t-s)\left(\alpha \nabla u_{m}(s), \nabla u_{m}^{\prime}(t)\right) d s= & \frac{1}{2} \frac{d}{d t}\left[\left(g \square \nabla u_{m}\right)(t)\right. \\
& \left.-\left(\int_{0}^{t} g(s) d s\right)\left|\sqrt{\alpha} \nabla u_{m}(t)\right|_{2}^{2}\right] \\
& +\frac{1}{2} g(t)\left|\sqrt{\alpha} \nabla u_{m}(t)\right|_{2}^{2} \\
& -\frac{1}{2}\left(g^{\prime} \square \nabla u_{m}\right)(t) .
\end{aligned}
$$

De (3.4) y (3.5), se obtiene

$$
\begin{aligned}
\frac{d}{d t} E(t)= & \left(f\left(u_{m}\right), u_{m}^{\prime}(t)\right)+\left(h\left(u_{m}\right), u_{m}^{\prime}(t)\right)_{\Gamma_{1}} \\
& -\frac{1}{2} g(t)\left|\sqrt{\alpha} \nabla u_{m}(t)\right|_{2}^{2}+\frac{1}{2}\left(g^{\prime} \square \nabla u_{m}\right)(t),
\end{aligned}
$$

donde

$$
\begin{aligned}
E(t)= & \frac{1}{2}\left|u_{m}^{\prime}(t)\right|_{2}^{2}+\frac{1}{2} \int_{\Omega} \alpha_{g}(x, t)\left|\nabla u_{m}(t)\right|^{2} d x \\
& +\int_{0}^{t}\left|\sqrt{\beta} u_{m}^{\prime}(s)\right|_{2}^{2} d s+\int_{0}^{t}\left|\sqrt{\delta} u_{m}^{\prime}(s)\right|_{2, \Gamma_{1}}^{2} d s+\frac{1}{2}\left(g \square \nabla u_{m}\right)(t), \\
\alpha_{g}(x, t)= & k_{0}-\alpha(x) \int_{0}^{t} g(\tau) d \tau .
\end{aligned}
$$

Observar que $\alpha_{g}(x, t) \geq l$, donde $l$ es dado en $\left(H_{2}\right)$. Ahora, usando la hipótesis $\left(H_{3}\right)$, la desigualdad de Hölder, la desigualdad de Sobolev-Poincaré y la desigualdad $a b \leq \varepsilon a^{2}+\frac{1}{4 \varepsilon} b^{2}, \forall a, b \geq 0$, $\forall \varepsilon>0$, se obtienen

$$
\begin{aligned}
\left(f\left(u_{m}\right), u_{m}^{\prime}(t)\right) & \leq N_{0} \int_{\Omega}\left|u_{m}(t)\right|^{p+1}\left|u_{m}^{\prime}(t)\right| d x \\
& \leq N_{0}\left|u_{m}(t)\right|_{2(p+1)}^{p+1}\left|u_{m}^{\prime}(t)\right|_{2} \\
& \leq N_{0} B_{1}^{p+1}\left\|u_{m}(t)\right\|^{p+1}\left|u_{m}^{\prime}(t)\right|_{2}
\end{aligned}
$$

y

$$
\begin{aligned}
\left(h\left(u_{m}\right), u_{m}^{\prime}(t)\right)_{\Gamma_{1}} & \leq M_{0} \int_{\Gamma_{1}}\left|u_{m}(t)\right|^{q+1}\left|u_{m}^{\prime}(t)\right| d \Gamma \\
& \leq M_{0}\left|u_{m}(t)\right|_{2(q+1), \Gamma_{1}}^{q+1}\left|u_{m}^{\prime}(t)\right|_{2, \Gamma_{1}} \\
& \leq M_{0} B_{2}^{q+1}\left\|u_{m}(t)\right\|^{q+1}\left|u_{m}^{\prime}(t)\right|_{2, \Gamma_{1}} \\
& \leq \frac{1}{4 \varepsilon} M_{0}^{2} B_{2}^{2(q+1)}\left\|u_{m}(t)\right\|^{2(q+1)}+\varepsilon\left|u_{m}^{\prime}(t)\right|_{2, \Gamma_{1}}^{2} .
\end{aligned}
$$

Sustituyendo (3.8) y (3.9) en (3.6), tomando $\varepsilon=\frac{\delta_{0}}{2}$ y utilizando (3.3), se obtiene

$$
\varphi_{m}(t) \leq C+C \int_{0}^{t}\left[\varphi_{m}^{\frac{p+2}{2}}(s)+\varphi_{m}^{q+1}(s)\right] d s
$$


donde

$$
\varphi_{m}(t)=\left|u_{m}^{\prime}(t)\right|_{2}^{2}+\left\|u_{m}(t)\right\|^{2}+\int_{0}^{t}\left|u_{m}^{\prime}(s)\right|_{2, \Gamma_{1}}^{2} d s
$$

y $C$ es una constante positiva independiente de $m$ y $t$.

Definamos la función

$$
G(\tau)=\int_{C}^{\tau} \frac{d s}{s^{\frac{p+2}{2}}+s^{q+1}}, \text { para } \tau \geq C .
$$

Entonces

$$
G(\infty) \leq \frac{1}{q C^{q}} \quad \text { o } \quad G(\infty) \leq \frac{2}{p C^{\frac{1}{p}}} .
$$

Por esta relación y utilizando la desigualdad generalizada de Gronwall, de (3.10), existe un número $\left.T_{0} \in\right] 0, G(\infty)\left[\right.$ y una constante $C_{1}>0$, independiente de $m$, tal que

$$
\left|u_{m}^{\prime}(t)\right|_{2}^{2}+\left\|u_{m}(t)\right\|^{2}+\int_{0}^{t}\left|u_{m}^{\prime}(s)\right|_{2, \Gamma_{1}}^{2} d s \leq C_{1},
$$

para todo $t \in\left[0, T_{0}\right]$ y para todo $m \in \mathbb{N}$.

Aplicando la hipótesis $\left(H_{3}\right)$, la desigualdad de Sobolev-Poincaré y (3.11), existe una constante $C_{2}>0$, independiente de $m$, tal que

$$
\left|f\left(u_{m}(t)\right)\right|_{2}^{2}+\left|h\left(u_{m}(t)\right)\right|_{2, \Gamma_{1}}^{2} \leq C_{2},
$$

para todo $t \in\left[0, T_{0}\right]$ y para todo $m \in \mathbb{N}$.

Segunda estimativa. En primer lugar vamos a estimar $u_{m}^{\prime \prime}(0)$ en la norma de $L^{2}(\Omega)$. Reemplazando $w$ por $u_{m}^{\prime \prime}(0)$ en (3.2) y considerando $t=0$, obtenemos

$$
\begin{aligned}
\left(u_{m}^{\prime \prime}(0), u_{m}^{\prime \prime}(0)\right) & -k_{0}\left(\Delta u_{m}(0), u_{m}^{\prime \prime}(0)\right)+\left(\frac{\partial u_{m}(0)}{\partial \nu}, u_{m}^{\prime \prime}(0)\right)_{\Gamma_{1}}+\left(\beta u_{m}^{\prime}(0), u_{m}^{\prime \prime}(0)\right) \\
+ & \left(\delta u_{m}^{\prime}(0), u_{m}^{\prime \prime}(0)\right)_{\Gamma_{1}}=\left(f\left(u_{m}(0)\right), u_{m}^{\prime \prime}(0)\right)+\left(h\left(u_{m}(0)\right), u_{m}^{\prime \prime}(0)\right)_{\Gamma_{1}}
\end{aligned}
$$

Del cual, se tiene

$$
\begin{aligned}
\left|u_{m}^{\prime \prime}(0)\right|_{2}^{2}= & \left(k_{0} \Delta u_{m}(0)-\beta u_{m}^{\prime}(0)+f\left(u_{m}(0)\right), u_{m}^{\prime \prime}(0)\right) \\
& +\left(h\left(u_{m}(0)\right)-\frac{\partial u_{m}(0)}{\partial \nu}-\delta u_{m}^{\prime}(0), u_{m}^{\prime \prime}(0)\right)_{\Gamma_{1}} .
\end{aligned}
$$

De esta igualdad, por (3.1) y ( 3.3$)$, existe una constante $C_{3}>0$, independiente de $m$, tal que

$$
\left|u_{m}^{\prime \prime}(0)\right|_{2} \leq C_{3}
$$

para todo $m \in \mathbb{N}$.

Ahora, diferenciando (3.2) con respecto de $t$ y sustituyendo $w$ por $u_{m}^{\prime \prime}(t)$, resulta

$$
\begin{aligned}
& \left(u_{m}^{\prime \prime \prime}(t), u_{m}^{\prime \prime}(t)\right)+k_{0}\left(\left(u_{m}^{\prime}(t), u_{m}^{\prime \prime}(t)\right)\right)+\left(\beta u_{m}^{\prime \prime}(t), u_{m}^{\prime \prime}(t)\right) \\
& -\int_{0}^{t} g^{\prime}(t-s)\left(\alpha \nabla u_{m}(s), \nabla u_{m}^{\prime \prime}(t)\right) d s \\
& -g(0)\left(\alpha \nabla u_{m}(t), \nabla u_{m}^{\prime \prime}(t)\right)+\left(\delta u_{m}^{\prime \prime}(t), u_{m}^{\prime \prime}(t)\right)_{\Gamma_{1}} \\
& \quad=\left(f^{\prime}\left(u_{m}\right) u_{m}^{\prime}(t), u_{m}^{\prime \prime}(t)\right)+\left(h^{\prime}\left(u_{m}\right) u_{m}^{\prime}(t), u_{m}^{\prime \prime}(t)\right)_{\Gamma_{1}} .
\end{aligned}
$$


Y utilizando las identidades

$$
\begin{aligned}
\int_{0}^{t} g^{\prime}(t-s)\left(\alpha \nabla u_{m}(s), \nabla u_{m}^{\prime \prime}(t)\right) d s= & \frac{d}{d t} \int_{0}^{t} g^{\prime}(t-s)\left(\alpha \nabla u_{m}(s), \nabla u_{m}^{\prime}(t)\right) d s \\
& -\int_{0}^{t} g^{\prime \prime}(t-s)\left(\alpha \nabla u_{m}(s), \nabla u_{m}^{\prime}(t)\right) d s \\
& -g^{\prime}(0)\left(\alpha \nabla u_{m}(t), \nabla u_{m}^{\prime}(t)\right)
\end{aligned}
$$

$\mathrm{y}$

$$
\begin{aligned}
\left(\alpha \nabla u_{m}(t), \nabla u_{m}^{\prime \prime}(t)\right)= & \frac{d}{d t}\left(\alpha \nabla u_{m}(t), \nabla u_{m}^{\prime}(t)\right) \\
& -\left|\sqrt{\alpha} \nabla u_{m}^{\prime}(t)\right|_{2}^{2},
\end{aligned}
$$

se obtiene

$$
\begin{aligned}
\frac{1}{2} \frac{d}{d t}\left[\left|u_{m}^{\prime \prime}(t)\right|_{2}^{2}+k_{0}\left\|u_{m}^{\prime}(t)\right\|^{2}\right]+\left|\sqrt{\beta} u_{m}^{\prime \prime}(t)\right|_{2}^{2}+\left|\sqrt{\delta} u_{m}^{\prime \prime}(t)\right|_{2, \Gamma_{1}}^{2} \\
=\frac{d}{d t} \int_{0}^{t} g^{\prime}(t-s)\left(\alpha \nabla u_{m}(s), \nabla u_{m}^{\prime}(t)\right) d s \\
\quad-\int_{0}^{t} g^{\prime \prime}(t-s)\left(\alpha \nabla u_{m}(s), \nabla u_{m}^{\prime}(t)\right) d s-g^{\prime}(0)\left(\alpha \nabla u_{m}(t), \nabla u_{m}^{\prime}(t)\right) \\
\quad+g(0) \frac{d}{d t}\left(\alpha \nabla u_{m}(t), \nabla u_{m}^{\prime}(t)\right)-g(0)\left|\sqrt{\alpha} \nabla u_{m}^{\prime}(t)\right|_{2}^{2} \\
+\left(f^{\prime}\left(u_{m}\right) u_{m}^{\prime}(t), u_{m}^{\prime \prime}(t)\right)+\left(h^{\prime}\left(u_{m}\right) u_{m}^{\prime}(t), u_{m}^{\prime \prime}(t)\right)_{\Gamma_{1}} .
\end{aligned}
$$

Desde que $\frac{p}{2(p+1)}+\frac{1}{2(p+1)}+\frac{1}{2}=1$, la desigualdad de Hölder, la desigualdad de Sobolev-Poincaré, (3.11) y la desigualdad de Young, resulta

$$
\begin{aligned}
\left|\left(f^{\prime}\left(u_{m}\right) u_{m}^{\prime}(t), u_{m}^{\prime \prime}(t)\right)\right| & \leq N_{0}\left|u_{m}(t)\right|_{2(p+1)}^{p}\left|u_{m}^{\prime}(t)\right|_{2(p+1)}\left|u_{m}^{\prime \prime}(t)\right|_{2} \\
& \leq N_{0} B_{1}^{p+1}\left\|u_{m}(t)\right\|\left\|^{p}\right\| u_{m}^{\prime}(t) \|\left|u_{m}^{\prime \prime}(t)\right|_{2} \\
& \leq N_{0} B_{1}^{p+1} C_{1}^{\frac{p}{2}}\left\|u_{m}^{\prime}(t)\right\|\left|u_{m}^{\prime \prime}(t)\right|_{2} \\
& \leq \frac{1}{4} N_{0}^{2} B_{1}^{2(p+1)} C_{1}^{p}\left\|u_{m}^{\prime}(t)\right\|^{2}+\left|u_{m}^{\prime \prime}(t)\right|_{2}^{2} \\
& =C_{1 N B}\left\|u_{m}^{\prime}(t)\right\|^{2}+\left|u_{m}^{\prime \prime}(t)\right|_{2}^{2},
\end{aligned}
$$

De manera análoga, se obtiene

$$
\left|\left(h^{\prime}\left(u_{m}\right) u_{m}^{\prime}(t), u_{m}^{\prime \prime}(t)\right)_{\Gamma_{1}}\right| \leq C_{2 M B}\left\|u_{m}^{\prime}(t)\right\|^{2}+\varepsilon\left|u_{m}^{\prime \prime}(t)\right|_{2, \Gamma_{1}}^{2}
$$

donde $\varepsilon>0$. Integrando (3.14) sobre el intervalo $(0, t)$ y teniendo en cuenta $(3.3),(3.13),(3.15)$ 
y (3.16), resulta

$$
\begin{aligned}
& \frac{1}{2}\left|u_{m}^{\prime \prime}(t)\right|_{2}^{2}+\frac{k_{0}}{2}\left\|u_{m}^{\prime}(t)\right\|^{2}+\int_{0}^{t}\left|\sqrt{\beta} u_{m}^{\prime \prime}(s)\right|_{2}^{2} d s+\int_{0}^{t}\left|\sqrt{\delta} u_{m}^{\prime \prime}(s)\right|_{2, \Gamma_{1}}^{2} d s \\
& \leq L_{0}+\int_{0}^{t} g^{\prime}(t-s)\left(\alpha \nabla u_{m}(s), \nabla u_{m}^{\prime}(t)\right) d s \\
&-\int_{0}^{t} \int_{0}^{\tau} g^{\prime \prime}(\tau-s)\left(\alpha \nabla u_{m}(s), \nabla u_{m}^{\prime}(\tau)\right) d s d \tau-g^{\prime}(0) \int_{0}^{t}\left(\alpha \nabla u_{m}(s), \nabla u_{m}^{\prime}(s)\right) d s \\
&+g(0)\left(\alpha \nabla u_{m}(t), \nabla u_{m}^{\prime}(t)\right)-g(0) \int_{0}^{t}\left|\sqrt{\alpha} \nabla u_{m}^{\prime}(s)\right|_{2}^{2} d s \\
&+C_{1 N B} \int_{0}^{t}\left\|u_{m}^{\prime}(s)\right\|^{2} d s+\int_{0}^{t}\left|u_{m}^{\prime \prime}(s)\right|_{2}^{2} d s \\
&+C_{2 M B} \int_{0}^{t}\left\|u_{m}^{\prime}(s)\right\|^{2} d s+\varepsilon \int_{0}^{t}\left|u_{m}^{\prime \prime}(s)\right|_{2, \Gamma_{1}}^{2} d s
\end{aligned}
$$

donde $L_{0}>0$ es una constante independiente de $m$ y $t$. Estimando, cinco términos de la derecha de (3.17), se obtienen

$$
\begin{gathered}
\int_{0}^{t} g^{\prime}(t-s)\left(\alpha \nabla u_{m}(s), \nabla u_{m}^{\prime}(t)\right) d s \\
\leq \xi_{1}\|\alpha\|_{L^{\infty}(\Omega)} \int_{0}^{t} g(t-s)\left\|u_{m}(s)\right\|\left\|u_{m}^{\prime}(t)\right\| d s \\
\leq \frac{\xi_{1}^{2}\|\alpha\|_{L^{\infty}(\Omega)}^{2}\|g\|_{L^{1}}}{4 \eta} \int_{0}^{t} g(t-s)\left\|u_{m}(s)\right\|^{2} d s+\eta\left\|u_{m}^{\prime}(t)\right\|^{2}, \\
-\int_{0}^{t} \int_{0}^{\tau} g^{\prime \prime}(\tau-s)\left(\alpha \nabla u_{m}(s), \nabla u_{m}^{\prime}(\tau)\right) d s d \tau \\
\leq \xi_{3}\|\alpha\|_{L^{\infty}(\Omega)} \int_{0}^{t} \int_{0}^{\tau} g(\tau-s)\left\|u_{m}(s)\right\|\left\|u_{m}^{\prime}(\tau)\right\| d s d \tau \\
\leq \int_{0}^{t}\left[\left\|u_{m}^{\prime}(\tau)\right\| \xi_{3}\|\alpha\|_{L^{\infty}(\Omega)} \int_{0}^{\tau} g(\tau-s)\left\|u_{m}(s)\right\| d s\right] d \tau \\
\leq \frac{\xi_{3}^{2}\|\alpha\|_{L^{\infty}(\Omega)}^{2} \int_{0}^{t}\left(\int_{0}^{\tau} g(\tau-s)\left\|u_{m}(s)\right\| d s\right)^{2} d \tau+\eta \int_{0}^{t}\left\|u_{m}^{\prime}(\tau)\right\|^{2} d \tau,}{4 \eta} \\
\int_{0}^{t}\left(\alpha \nabla u_{m}(s), \nabla u_{m}^{\prime}(s)\right) d s \leq \frac{\left|g^{\prime}(0)\right|^{2}\|\alpha\|_{L^{\infty}(\Omega)}^{2}}{4} \int_{0}^{t}\left\|u_{m}(s)\right\|^{2} d s+\int_{0}^{t}\left\|u_{m}^{\prime}(s)\right\|^{2} d s, \\
g(0)\left(\alpha \nabla u_{m}(t), \nabla u_{m}^{\prime}(t)\right) \leq \frac{1}{4 \eta} g^{2}(0)\|\alpha\|_{L^{\infty}(\Omega)}^{2}\left\|u_{m}(t)\right\|^{2}+\eta\left\|u_{m}^{\prime}(t)\right\|^{2}
\end{gathered}
$$

y

$$
-g(0) \int_{0}^{t}\left|\sqrt{\alpha} \nabla u_{m}^{\prime}(s)\right|_{2}^{2} d s \leq g(0)\|\alpha\|_{L^{\infty}(\Omega)} \int_{0}^{t}\left\|u_{m}^{\prime}(s)\right\|^{2} d s .
$$

Sustituyendo estas últimas cinco estimativas en (3.17), tomando $\varepsilon=\frac{\delta_{0}}{2}$ y $\eta=\frac{k_{0}}{8}$, utilizando la estimativa $(3.11)$ y las hipótesis $\left(H_{1}\right)$ y $\left(H_{2}\right)$, se obtiene

$$
\psi_{m}(t) \leq C_{*}+C_{*} \int_{0}^{t} \psi_{m}(s) d s
$$


donde

$$
\psi_{m}(t)=\left|u_{m}^{\prime \prime}(t)\right|_{2}^{2}+\left\|u_{m}^{\prime}(t)\right\|^{2}+\int_{0}^{t}\left|u_{m}^{\prime \prime}(s)\right|_{2, \Gamma_{1}}^{2} d s
$$

y $C_{*}$ es una constante positiva independiente de $m$ y depende de $T_{0}$. Utilizando la desigualdad de Gronwall en (3.18), existe una constante $C_{4}>0$, independiente de $m$, tal que

$$
\left|u_{m}^{\prime \prime}(t)\right|_{2}^{2}+\left\|u_{m}^{\prime}(t)\right\|^{2}+\int_{0}^{t}\left|u_{m}^{\prime \prime}(s)\right|_{2, \Gamma_{1}}^{2} d s \leq C_{4},
$$

para todo $t \in\left[0, T_{0}\right]$ y para todo $m \in \mathbb{N}$.

Pasaje al límite. De las estimativas (3.11) y (3.19), existen subsucesiones $\left(u_{\nu}\right)_{\nu \in \mathbb{N}},\left(u_{\nu}^{\prime}\right)_{\nu \in \mathbb{N}} \mathrm{y}$ $\left(u_{\nu}^{\prime \prime}\right)_{\nu \in \mathbb{N}}$ de $\left(u_{k}\right)_{k \in \mathbb{N}},\left(u_{k}^{\prime}\right)_{k \in \mathbb{N}} \mathrm{y}\left(u_{k}^{\prime \prime}\right)_{k \in \mathbb{N}}$, respectivamente, tales que

$$
\begin{aligned}
& u_{\nu} \rightarrow u \quad \text { débil estrella en } L^{\infty}\left(0, T_{0} ; H_{\Gamma_{0}}^{1}(\Omega)\right), \\
& u_{\nu}^{\prime} \rightarrow u^{\prime} \quad \text { débil estrella en } L^{\infty}\left(0, T_{0} ; H_{\Gamma_{0}}^{1}(\Omega)\right) \text {, } \\
& u_{\nu}^{\prime \prime} \rightarrow u^{\prime \prime} \text { débil estrella en } L^{\infty}\left(0, T_{0} ; L^{2}(\Omega)\right) \text {, } \\
& u_{\nu}^{\prime} \rightarrow u^{\prime} \quad \text { débil en } \quad L^{2}\left(0, T_{0} ; L^{2}\left(\Gamma_{1}\right)\right), \\
& u_{\nu}^{\prime \prime} \rightarrow u^{\prime \prime} \text { débil en } L^{2}\left(0, T_{0} ; L^{2}\left(\Gamma_{1}\right)\right) .
\end{aligned}
$$

Desde que se verifica las inmersiones compactas $H_{\Gamma_{0}}^{1}(\Omega) \stackrel{c}{\hookrightarrow} L^{2}(\Omega)$ y $H^{\frac{1}{2}}\left(\Gamma_{1}\right) \stackrel{c}{\hookrightarrow} L^{2}\left(\Gamma_{1}\right)$, por el Lema de compacidad de Lions-Aubin, se tiene

$$
\begin{array}{lll}
u_{\nu} \rightarrow u & \text { fuerte en } & L^{2}\left(0, T_{0} ; L^{2}(\Omega)\right), \\
u_{\nu}^{\prime} \rightarrow u^{\prime} & \text { fuerte en } & L^{2}\left(0, T_{0} ; L^{2}(\Omega)\right), \\
u_{\nu} \rightarrow u & \text { fuerte en } & L^{2}\left(0, T_{0} ; L^{2}\left(\Gamma_{1}\right)\right)
\end{array}
$$

y consecuentemente por las continuidades de $f$ y $h$, aplicando el Lema de Lions, se obtiene

$$
\begin{aligned}
& f\left(u_{\nu}\right) \rightarrow f(u) \quad \text { débil en } \quad L^{2}\left(0, T_{0} ; L^{2}(\Omega)\right), \\
& h\left(u_{\nu}\right) \rightarrow h(u) \quad \text { débil en } \quad L^{2}\left(0, T_{0} ; L^{2}\left(\Gamma_{1}\right)\right) .
\end{aligned}
$$

Por otra parte, de la estimativa (3.12), resulta

$$
\begin{aligned}
& f\left(u_{\nu}\right) \rightarrow \chi_{1} \quad \text { débil estrella en } L^{\infty}\left(0, T_{0} ; L^{2}(\Omega)\right), \\
& h\left(u_{\nu}\right) \rightarrow \chi_{2} \text { débil estrella en } L^{\infty}\left(0, T_{0} ; L^{2}\left(\Gamma_{1}\right)\right) \text {. }
\end{aligned}
$$

De aquí y (3.22), implica

$$
\begin{aligned}
& f\left(u_{\nu}\right) \rightarrow f(u) \quad \text { débil estrella en } \quad L^{\infty}\left(0, T_{0} ; L^{2}(\Omega)\right), \\
& h\left(u_{\nu}\right) \rightarrow h(u) \quad \text { débil estrella en } \quad L^{\infty}\left(0, T_{0} ; L^{2}\left(\Gamma_{1}\right)\right) .
\end{aligned}
$$

De las convergencias (3.20), (3.21) y (3.23), por pasaje al límite en la ecuación aproximada (3.2), resulta

$$
\begin{array}{r}
\frac{d}{d t}\left(u^{\prime}(t), w\right)-k_{0}(\Delta u(t), w)+\int_{0}^{t} g(t-s)(\operatorname{div}[\alpha(x) \nabla u(s)], w) d s+\left(\beta u^{\prime}(t), w\right) \\
=(f(u(t)), w),
\end{array}
$$

para todo $w \in H_{\Gamma_{0}}^{1}(\Omega)$, en el sentido de las distribuciones $\mathcal{D}^{\prime}\left(0, T_{0}\right)$. De aquí se obtiene

$$
\begin{aligned}
u^{\prime \prime}-k_{0} \Delta u+\int_{0}^{t} g(t-s) \operatorname{div}[\alpha(x) \nabla & u(s)] d s+\beta(x) u^{\prime} \\
& =f(u) \text { en } \mathcal{D}^{\prime}\left(\Omega \times\left(0, T_{0}\right)\right) .
\end{aligned}
$$


Desde que $\beta(x) u^{\prime} \in L^{\infty}\left(0, T_{0} ; H_{\Gamma_{0}}^{1}(\Omega)\right), u^{\prime \prime} \in L^{\infty}\left(0, T_{0} ; L^{2}(\Omega)\right), f(u) \in L^{\infty}\left(0, T_{0}, L^{2}(\Omega)\right)$ y de la inmersión continua

$$
H_{\Gamma_{0}}^{1}(\Omega) \hookrightarrow L^{2}(\Omega),
$$

resulta

$$
-k_{0} \Delta u+\int_{0}^{t} g(t-s) \operatorname{div}[\alpha(x) \nabla u(s)] d s \in L^{\infty}\left(0, T_{0} ; L^{2}(\Omega)\right) .
$$

Por tanto, de (3.24) se obtiene

$$
u^{\prime \prime}-k_{0} \Delta u+\int_{0}^{t} g(t-s) \operatorname{div}[\alpha(x) \nabla u(s)] d s+\beta(x) u^{\prime}=f(u) \quad \text { en } \quad L^{\infty}\left(0, T_{0} ; L^{2}(\Omega)\right) .
$$

Considerando (3.25) y usando el teorema de la divergencia, se deduce

$$
k_{0} \frac{\partial u}{\partial \nu}-\int_{0}^{t} g(t-s)[\alpha(x) \nabla u(s)] \cdot \nu d s+\delta(x) u^{\prime}=h(u) \quad \text { en } \mathcal{D}^{\prime}\left(0, T ; H^{-\frac{1}{2}}\left(\Gamma_{1}\right)\right)
$$

y desde que $h(u) \in L^{\infty}\left(0, T_{0}, L^{2}\left(\Gamma_{1}\right)\right)$, resulta

$$
k_{0} \frac{\partial u}{\partial \nu}-\int_{0}^{t} g(t-s)[\alpha(x) \nabla u(s)] \cdot \nu d s+\delta(x) u^{\prime}=h(u) \quad \text { en } \quad L^{\infty}\left(0, T_{0} ; L^{2}\left(\Gamma_{1}\right)\right) .
$$

Denotemos

$$
A u=-k_{0} \Delta u+g * \operatorname{div}[\alpha(x) \nabla u] .
$$

Entonces para casi todo $t \in] 0, T_{0}\left[, A u(t) \in L^{2}(\Omega)\right.$. Por el Teorema de regularidad de los problemas elípticos, para casi todo $t \in] 0, T_{0}\left[\right.$, se tiene $u(t) \in H^{2}(\Omega)$. Además

$$
\|u(t)\|_{H^{2}(\Omega)} \leq C\left[\left|u^{\prime \prime}(t)\right|_{2}+\left|\beta u^{\prime}(t)\right|_{2}+|f(u(t))|_{2}\right] .
$$

Desde que las funciones del lado derecho en esta última desigualdad pertenecen al espacio $L^{\infty}\left(0, T_{0}\right)$, resulta $u \in L^{\infty}\left(0, T_{0}, H_{\Gamma_{0}}^{1}(\Omega) \cap H^{2}(\Omega)\right)$. Con esto se concluye la existencia de la solución fuerte del problema (1.1) - (1.4). A continuación, pasemos a probar la unicidad de solución del problema.

Unicidad de solución. Sean $u$ y $v$ soluciones fuertes del problema (1.1) - (1.4). Considerando $z=u-v$, resulta que $z$ satisface

$$
\begin{aligned}
\left(z^{\prime \prime}(t), w\right)+k_{0}((z(t), w)) & +\left(\beta z^{\prime}, w\right)-\int_{0}^{t} g(t-s)(\alpha \nabla z(s), \nabla w) d s \\
& +\left(\delta z^{\prime}, w\right)_{\Gamma_{1}}=(f(u)-f(v), w)+(h(u)-h(v), w)_{\Gamma_{1}}
\end{aligned}
$$

para todo $w \in H_{\Gamma_{0}}^{1}(\Omega)$.

Sustituyendo $w=z^{\prime}(t)$ en (3.27), implica

$$
\begin{aligned}
\frac{1}{2} \frac{d}{d t}\left|z^{\prime}(t)\right|_{2}^{2}+ & \frac{k_{0}}{2} \frac{d}{d t}\|z(t)\|^{2}+\left|\sqrt{\beta} z^{\prime}(t)\right|_{2}^{2} \\
-\int_{0}^{t} g(t-s) & \left(\alpha \nabla z(s), \nabla z^{\prime}(t)\right) d s+\left|\sqrt{\delta} z^{\prime}(t)\right|_{2, \Gamma_{1}}^{2} \\
& =\left(f(u)-f(v), z^{\prime}(t)\right)+\left(h(u)-h(v), z^{\prime}(t)\right)_{\Gamma_{1}}
\end{aligned}
$$


y utilizando la identidad

$$
\begin{aligned}
-\int_{0}^{t} g(t-s)\left(\alpha \nabla z(s), \nabla z^{\prime}(t)\right) d s= & \frac{1}{2} \frac{d}{d t}[(g \square \nabla z)(t) \\
& \left.-\left(\int_{0}^{t} g(s) d s\right)|\sqrt{\alpha} \nabla z(t)|_{2}^{2}\right] \\
& +\frac{1}{2} g(t)|\sqrt{\alpha} \nabla z(t)|_{2}^{2} \\
& -\frac{1}{2}\left(g^{\prime} \square \nabla z\right)(t) .
\end{aligned}
$$

se obtiene

$$
\begin{aligned}
\frac{d}{d t}\left[\frac{1}{2}\left|z^{\prime}(t)\right|_{2}^{2}+\frac{1}{2} \int_{\Omega} \alpha_{g}(x, t)|\nabla z(t)|^{2} d x+\frac{1}{2}(g \square \nabla z)(t)\right] \\
+\left|\sqrt{\beta} z^{\prime}(t)\right|_{2}^{2}+\left|\sqrt{\delta} z^{\prime}(t)\right|_{2, \Gamma_{1}}^{2}+\frac{1}{2} g(t)|\sqrt{\alpha} \nabla z(t)|_{2}^{2}-\frac{1}{2}\left(g^{\prime} \square \nabla z\right)(t) \\
=\left(f(u)-f(v), z^{\prime}(t)\right)+\left(h(u)-h(v), z^{\prime}(t)\right)_{\Gamma_{1}} .
\end{aligned}
$$

Usando la hipótesis $\left(H_{3}\right)$, la desigualdad de Hölder, la desigualdad de Sobolev-Poincaré y la desigualdad $a b \leq \frac{1}{4 \varepsilon} a^{2}+\varepsilon b^{2}, \forall a, b \geq 0, \forall \varepsilon>0$, se obtiene

$$
\begin{aligned}
\left(f(u)-f(v), z^{\prime}(t)\right) & \leq N_{0} \int_{\Omega}\left(|u(t)|^{p}+|v(t)|^{p}\right)|z(t)|\left|z^{\prime}(t)\right| d x \\
& \leq N_{0}\left(|u(t)|_{2(p+1)}^{p}+|v(t)|_{2(p+1)}^{p}\right)|z(t)|_{2(p+1)}\left|z^{\prime}(t)\right|_{2} \\
& \leq N_{1}\left(\|u(t)\|^{p}+\|v(t)\|^{p}\right)\|z(t)\|\left|z^{\prime}(t)\right|_{2} \\
& \leq \frac{N_{1}^{2}}{2}\left(\|u(t)\|^{2 p}+\|v(t)\|^{2 p}\right)\|z(t)\|^{2}+\frac{1}{2}\left|z^{\prime}(t)\right|_{2}^{2}
\end{aligned}
$$

y

$$
\begin{aligned}
\left(h(u)-h(v), z^{\prime}(t)\right)_{\Gamma_{1}} & \leq M_{0} \int_{\Gamma_{1}}\left(|u(t)|^{q}+|v(t)|^{q}\right)|z(t)|\left|z^{\prime}(t)\right| d \Gamma \\
& \leq M_{0}\left(|u(t)|_{2(q+1), \Gamma_{1}}^{q}+|v(t)|_{2(q+1), \Gamma_{1}}^{q}\right)|z(t)|_{2(q+1), \Gamma_{1}}\left|z^{\prime}(t)\right|_{2, \Gamma_{1}} \\
& \leq M_{1}\left(\|u(t)\|^{q}+\|v(t)\|^{q}\right)\|z(t)\|\left|z^{\prime}(t)\right|_{2, \Gamma_{1}} \\
& \leq \frac{1}{4 \varepsilon} M_{1}^{2}\left(\|u(t)\|^{2 q}+\|v(t)\|^{2 q}\right)\|z(t)\|^{2}+\varepsilon\left|z^{\prime}(t)\right|_{2, \Gamma_{1}}^{2} .
\end{aligned}
$$

Desde que $u, v \in L^{\infty}\left(0, T ; H_{\Gamma_{0}}^{1}(\Omega)\right)$, sustituyendo (3.29) y (3.30) en (3.28), tomando $\varepsilon=\frac{\delta_{0}}{2}$, se obtiene

$$
\left|z^{\prime}(t)\right|_{2}^{2}+\|z(t)\|^{2} \leq C \int_{0}^{t}\left[\left|z^{\prime}(s)\right|_{2}^{2}+\|z(s)\|^{2}\right] d s,
$$

donde $C$ es una constante positiva. Aplicando el lema de Gronwall, resulta $z(t)=0$. Por tanto $u=v$, es decir que la solución fuerte es única. Los datos iniciales se verifican de modo estándar. Utilizando argumentos estándar de densidad con problemas truncados, se obtiene la solución débil del problema (1.1) - (1.4). Las discusiones son similares como en [11]. Esto completa la demostración del Teorema 3.1.

\section{No Existencia Global}


En esta sección, discutiremos la propiedad de explosión de soluciones en tiempo finito del problema (1.1) - (1.4). En la discusión emplearemos el método directo de la energía, utilizado por Li y Tsai $[6]$.

La energía del problema (1.1) - (1.4) se define por

$$
\begin{aligned}
E(t)= & \frac{1}{2}\left|u^{\prime}(t)\right|_{2}^{2}+\frac{1}{2} \int_{\Omega} \alpha_{g}(x, t)|\nabla u(x, t)|^{2} d x+\frac{1}{2}(g \square \nabla u)(t) \\
& -\int_{\Omega} F(u(t)) d x-\int_{\Gamma_{1}} H(u(t)) d \Gamma,
\end{aligned}
$$

para $t \geq 0$, donde

$$
\alpha_{g}(x, t)=k_{0}-\alpha(x) \int_{0}^{t} g(\tau) d \tau, \quad F(s)=\int_{0}^{s} f(\xi) d \xi \quad \text { y } H(s)=\int_{0}^{s} h(\xi) d \xi .
$$

Lema 3.2. La energía $E(t)$ es una función no creciente para $t \geq 0$,

$$
E^{\prime}(t)=-\left|\sqrt{\beta} u^{\prime}(t)\right|_{2}^{2}-\left|\sqrt{\delta} u^{\prime}(t)\right|_{2, \Gamma_{1}}^{2}-\frac{1}{2} g(t)|\sqrt{\alpha} \nabla u(t)|_{2}^{2}+\frac{1}{2}\left(g^{\prime} \square \nabla u\right)(t) \leq 0
$$

$y$

$$
E(t)+\int_{0}^{t}\left|\sqrt{\beta} u^{\prime}(s)\right|_{2}^{2} d s+\int_{0}^{t}\left|\sqrt{\delta} u^{\prime}(s)\right|_{2, \Gamma_{1}}^{2} d s \leq E(0)
$$

donde

$$
E(0)=\frac{1}{2}\left|u_{1}\right|_{2}^{2}+\frac{k_{0}}{2}\left\|u_{0}\right\|^{2}-\int_{\Omega} F\left(u_{0}(x)\right) d x-\int_{\Gamma_{1}} H\left(u_{0}\right) d \Gamma .
$$

Demostración. Multiplicando (1.1) por $u^{\prime}$, integrando sobre $\Omega$ y utilizando (1.2) - (1.4), se obtiene (3.32). La desigualdad (3.33) es una consecuencia directa de (3.32).

Lema 3.3. Supongamos que se verifica las hipótesis $\left(H_{1}\right)-\left(H_{4}\right)$. Si u es una solución del problema (1.1) - (1.4) con datos iniciales $u_{0} \in H_{\Gamma_{0}}^{1}(\Omega)$ y $u_{1} \in L^{2}(\Omega)$, entonces

$$
\begin{aligned}
A^{\prime \prime}(t)-4(\gamma+1)[ & \left|u^{\prime}(t)\right|_{2}^{2}+\int_{0}^{t}\left|\sqrt{\beta} u^{\prime}(s)\right|_{2}^{2} d s \\
& \left.+\int_{0}^{t}\left|\sqrt{\delta} u^{\prime}(s)\right|_{2, \Gamma_{1}}^{2} d s\right] \geq-4(2 \gamma+1) E(0),
\end{aligned}
$$

donde

$$
A(t)=|u(t)|_{2}^{2}+\int_{0}^{t}|\sqrt{\beta} u(s)|_{2}^{2} d s+\int_{0}^{t}|\sqrt{\delta} u(s)|_{2, \Gamma_{1}}^{2} d s
$$

Demostración. De (3.35), resulta

$$
A^{\prime}(t)=2\left(u(t), u^{\prime}(t)\right)+|\sqrt{\beta} u(t)|_{2}^{2}+|\sqrt{\delta} u(t)|_{2, \Gamma_{1}}^{2}
$$

$\mathrm{y}$

$$
\begin{aligned}
A^{\prime \prime}(t)= & 2\left|u^{\prime}(t)\right|_{2}^{2}-2 k_{0}\|u(t)\|^{2}+2 \int_{0}^{t} g(t-s)(\alpha \nabla u(s), \nabla u(t)) d s \\
& +2(f(u(t)), u(t))+2(h(u(t)), u(t))_{\Gamma_{1}} .
\end{aligned}
$$


Por la desigualdad de Young, obtenemos

$$
\begin{aligned}
2 \int_{0}^{t} g(t-s)(\alpha \nabla u(s), \nabla u(t)) d s= & 2 \int_{0}^{t} g(t-s)(\sqrt{\alpha}(\nabla u(s)-\nabla u(t)), \sqrt{\alpha} \nabla u(t)) d s \\
& +2\left(\int_{0}^{t} g(s) d s\right)|\sqrt{\alpha} \nabla u(t)|_{2}^{2} \\
\geq & -\int_{0}^{t} g(t-s)|\sqrt{\alpha}(\nabla u(s)-\nabla u(t))|_{2}^{2} d s \\
& -\int_{0}^{t} g(t-s)|\sqrt{\alpha} \nabla u(t)|_{2}^{2} d s \\
& +2\left(\int_{0}^{t} g(s) d s\right)|\sqrt{\alpha} \nabla u(t)|_{2}^{2} \\
= & \left(\int_{0}^{t} g(s) d s\right)|\sqrt{\alpha} \nabla u(t)|_{2}^{2}-(g \square \nabla u)(t) .
\end{aligned}
$$

Por (3.33), (3.37) y (3.38), se obtiene

$$
\begin{aligned}
A^{\prime \prime}(t)- & 4(\gamma+1)\left[\left|u^{\prime}(t)\right|_{2}^{2}+\int_{0}^{t}\left|\sqrt{\beta} u^{\prime}(s)\right|_{2}^{2} d s+\int_{0}^{t}\left|\sqrt{\delta} u^{\prime}(s)\right|_{2, \Gamma_{1}}^{2} d s\right] \\
\geq & -4(2 \gamma+1) E(0)+4 k_{0} \gamma\|u(t)\|^{2}-(4 \gamma+1)\left(\int_{0}^{t} g(s) d s\right)|\sqrt{\alpha} \nabla u(t)|_{2}^{2} \\
& +(4 \gamma+1)(g \square \nabla u)(t) \\
& +2\left[\int_{\Omega}(u f(u)-2(2 \gamma+1) F(u)) d x+\int_{\Gamma_{1}}(u h(u)-2(2 \gamma+1) H(u)) d x\right] \\
& +4 \gamma\left[\int_{0}^{t}\left|\sqrt{\beta} u^{\prime}(s)\right|_{2}^{2} d s+\int_{0}^{t}\left|\sqrt{\delta} u^{\prime}(s)\right|_{2, \Gamma_{1}}^{2} d s\right] .
\end{aligned}
$$

De aquí y utilizando $\gamma \geq \frac{k_{0}-l}{4 l}$, se obtiene (3.34).

Lema 3.4. Supongamos que se verifica las hipótesis $\left(H_{1}\right)-\left(H_{4}\right)$. Si u es una solución del problema (1.1) - (1.4) con datos iniciales $u_{0} \in H_{\Gamma_{0}}^{1}(\Omega)$ y $u_{1} \in L^{2}(\Omega)$, y satisfaciendo una de las siguientes condiciones:

(i) $E(0)<0$,

(ii) $E(0)=0$ y $A^{\prime}(0)>K_{0}$,

(iii) $E(0)>0$ y $A^{\prime}(0)>r_{2}\left[A(0)+\frac{K_{1}}{4(\gamma+1)}\right]+K_{0}$,

donde

$$
\begin{gathered}
K_{0}=\left|\sqrt{\beta} u_{0}\right|_{2}^{2}+\left|\sqrt{\delta} u_{0}\right|_{2, \Gamma_{1}}^{2}, \\
A(0)=\left|u_{0}\right|_{2}^{2}, \quad A^{\prime}(0)=2\left(u_{0}, u_{1}\right)+K_{0}, \\
K_{1}=4(\gamma+1) K_{0}+4(2 \gamma+1) E(0), \\
r_{2}=2(\gamma+1)-2 \sqrt{\gamma(\gamma+1)},
\end{gathered}
$$

entonces

$$
A^{\prime}(t)>K_{0} \text {, para } t>t_{0},
$$

donde $t_{0}=\operatorname{máx}\left\{\frac{A^{\prime}(0)-K_{0}}{4(2 \gamma+1) E(0)}, 0\right\}$ en el caso $(i)$ y $t_{0}=0$ en los casos $(i i)$ y (iii). 
Demostración. Consideremos tres casos de acuerdo al signo de la energía inicial $E(0)$ :

(i) Si $E(0)<0$, entonces de $(3.34)$, resulta

$$
A^{\prime}(t) \geq A^{\prime}(0)-4(2 \gamma+1) E(0) t .
$$

Considerando $A^{\prime}(0)-K_{0}-4(2 \gamma+1) E(0) t>0$, obtenemos

$$
A^{\prime}(t)>K_{0} \text {, para } t>t_{0},
$$

donde

$$
t_{0}=\operatorname{máx}\left\{\frac{A^{\prime}(0)-K_{0}}{4(2 \gamma+1) E(0)}, 0\right\} .
$$

(ii) Si $E(0)=0$, entonces de (3.34), resulta

$$
A^{\prime}(t) \geq A^{\prime}(0)
$$

Considerando $A^{\prime}(0)-K_{0}>0$, obtenemos

$$
A^{\prime}(t)>K_{0}, \text { para } t>0 .
$$

(iii) Si $E(0)>0$. Primero notemos que se cumple

$$
2 \int_{0}^{t}\left(\sqrt{\beta} u^{\prime}(s), \sqrt{\beta} u(s)\right) d s=|\sqrt{\beta} u(t)|_{2}^{2}-\left|\sqrt{\beta} u_{0}\right|_{2}^{2}
$$

$\mathrm{y}$

$$
2 \int_{0}^{t}\left(\sqrt{\delta} u^{\prime}(s), \sqrt{\delta} u(s)\right)_{\Gamma_{1}} d s=|\sqrt{\delta} u(t)|_{2, \Gamma_{1}}^{2}-\left|\sqrt{\delta} u_{0}\right|_{2, \Gamma_{1}}^{2} .
$$

De estos dos resultados, por la desigualdad de Hölder y la desigualdad de Young, resulta

$$
|\sqrt{\beta} u(t)|_{2}^{2} \leq\left|\sqrt{\beta} u_{0}\right|_{2}^{2}+\int_{0}^{t}|\sqrt{\beta} u(s)|_{2}^{2} d s+\int_{0}^{t}\left|\sqrt{\beta} u^{\prime}(s)\right|_{2}^{2} d s
$$

y

$$
|\sqrt{\delta} u(t)|_{2, \Gamma_{1}}^{2} \leq\left|\sqrt{\delta} u_{0}\right|_{2, \Gamma_{1}}^{2}+\int_{0}^{t}|\sqrt{\delta} u(s)|_{2, \Gamma_{1}}^{2} d s+\int_{0}^{t}\left|\sqrt{\delta} u^{\prime}(s)\right|_{2, \Gamma_{1}}^{2} d s
$$

Ahora, usando la desigualdad de Hölder y la desigualdad de Young en (3.36) y por (3.42) - (3.43), implica

$$
A^{\prime}(t) \leq A(t)+K_{0}+\left|u^{\prime}(t)\right|_{2}^{2}+\int_{0}^{t}\left|\sqrt{\beta} u^{\prime}(s)\right|_{2}^{2} d s+\int_{0}^{t}\left|\sqrt{\delta} u^{\prime}(s)\right|_{2, \Gamma_{1}}^{2} d s
$$

Empleando (3.34) y (3.44), se obtiene

$$
A^{\prime \prime}(t)-4(\gamma+1) A^{\prime}(t)+4(\gamma+1) A(t)+K_{1} \geq 0,
$$

donde

$$
K_{1}=4(\gamma+1) K_{0}+4(2 \gamma+1) E(0) .
$$

Definamos la función

$$
B(t)=A(t)+\frac{K_{1}}{4(\gamma+1)}
$$


Entonces $B(t)$ satisface las condiciones del Lema 2.5 . Por tanto, si

$$
A^{\prime}(0) \geq r_{2}\left(A(0)+\frac{K_{1}}{4(\gamma+1)}\right)+K_{0},
$$

entonces del Lema 2.5, deducimos que

$$
A^{\prime}(t)>K_{0}, \text { para } t>0 .
$$

Esto completa la demostración del Lema 3.4.

Teorema 3.5 (Explosión de soluciones). Supongamos que se verifica las hipótesis $\left(H_{1}\right)-$ $\left(H_{4}\right)$. Si u es una solución del problema (1.1) - (1.4) con datos iniciales $u_{0} \in H_{\Gamma_{0}}^{1}(\Omega)$ y $u_{1} \in L^{2}(\Omega)$, y satisfaciendo una de las siguientes condiciones:

(i) $E(0)<0$,

(ii) $E(0)=0$ y $A^{\prime}(0)>K_{0}$,

(iii) $0<E(0)<\frac{\left[A^{\prime}(0)-K_{0}\right]^{2}}{8\left[A(0)+T_{1} K_{0}\right]}$ y $A^{\prime}(0)>r_{2}\left[A(0)+\frac{K_{1}}{4(\gamma+1)}\right]+K_{0}$,

entonces la solución u explota en tiempo finito $T_{*}$ en el sentido de

$$
\lim _{t \rightarrow T_{*}^{-}}\|u(t)\|^{2}=\infty .
$$

Demostración. Consideremos la función

$$
J(t)=\left[A(t)+\left(T_{1}-t\right) K_{0}\right]^{-\gamma}, \text { para } t \in\left[0, T_{1}\right],
$$

donde $T_{1}>0$ es una constante que se determinará posteriormente y $\gamma$ es la constante dada en $\left(H_{4}\right)$. Tenemos

$$
J^{\prime}(t)=-\gamma[J(t)]^{\frac{1}{\gamma}+1}\left[A^{\prime}(t)-K_{0}\right]
$$

$\mathrm{y}$

$$
J^{\prime \prime}(t)=-\gamma[J(t)]^{\frac{2}{\gamma}+1} V(t),
$$

donde

$$
V(t)=A^{\prime \prime}(t)\left[A(t)+\left(T_{1}-t\right) K_{0}\right]-(\gamma+1)\left[A^{\prime}(t)-K_{0}\right]^{2} .
$$

Utilizando $(3.40)-(3.41)$, la desigualdad $\left(\sum_{i=1}^{3} a_{i} b_{i}\right)^{2} \leq\left(\sum_{i=1}^{3} a_{i}^{2}\right)\left(\sum_{i=1}^{3} b_{i}^{2}\right)$ y la desigualdad de Hölder, de (3.36), se obtiene

$$
\begin{aligned}
{\left[A^{\prime}(t)-K_{0}\right]^{2} \leq } & 4\left[A(t)+\left(T_{1}-t\right) K_{0}\right]\left[\left|u^{\prime}(t)\right|_{2}^{2}\right. \\
& \left.+\int_{0}^{t}\left|\sqrt{\beta} u^{\prime}(s)\right|_{2}^{2} d s+\int_{0}^{t}\left|\sqrt{\beta} u^{\prime}(s)\right|_{2, \Gamma_{1}}^{2} d s\right] .
\end{aligned}
$$

Por (3.48) y (3.49), resulta

$$
J^{\prime \prime}(t) \leq-\gamma[J(t)]^{\frac{1}{\gamma}+1} K(t),
$$

donde

$$
K(t)=A^{\prime \prime}(t)-4(\gamma+1)\left[\left|u^{\prime}(t)\right|_{2}^{2}+\int_{0}^{t}\left|\sqrt{\beta} u^{\prime}(s)\right|_{2}^{2} d s+\int_{0}^{t}\left|\sqrt{\beta} u^{\prime}(s)\right|_{2, \Gamma_{1}}^{2} d s\right] .
$$


De (3.34) y (3.50), se obtiene

$$
J^{\prime \prime}(t) \leq 4 \gamma(2 \gamma+1) E(0)[J(t)]^{\frac{1}{\gamma}+1}, \text { para } t \geq t_{0} .
$$

Utilizando (3.39) y (3.47), se tiene

$$
J^{\prime}(t)<0, \text { para } t>t_{0}
$$

Multiplicando (3.51) por $J^{\prime}(t)$ y luego integrando de $t_{0}$ a $t$, resulta

$$
\left[J^{\prime}(t)\right]^{2} \geq a+b[J(t)]^{2+\frac{1}{\gamma}}, \text { para } t \geq t_{0},
$$

donde

$$
a=\gamma^{2}\left[J\left(t_{0}\right)\right]^{\frac{2}{\gamma}+2}\left[\left[A^{\prime}\left(t_{0}\right)-K_{0}\right]^{2}-8 E(0)\left[J\left(t_{0}\right)\right]^{\frac{-1}{\gamma}}\right]
$$

$\mathrm{y}$

$$
b=8 \gamma^{2} E(0) .
$$

Entonces, por el Lema 2.6, existe un tiempo finito $T_{*}$ tal que

$$
\lim _{t \rightarrow T_{*}^{-}} J(t)=0 .
$$

Esto indica que $\operatorname{lím}_{t \rightarrow T_{*}^{-}}|u(t)|_{2}^{2}=\infty$. De aquí, usando la desigualdad de Sololev-Poincaré, se obtiene (3.45). Además se obtiene las estimativas para el tiempo finito de explosión $T_{*}$. Esto completa la demostración del Teorema 3.5.

Observación 3.6. La elección de la constante positiva $T_{1}$ en (3.46) se consigue con algunas consideraciones. Las discusiones son similares como en [8]. Omitimos los detalles.

\section{Conclusión}

En el presente estudio se ha obtenido: Existencia de la solución local mediante el método de Faedo-Galerkin y no existencia de la solución global utilizando el método indirecto con energía inicial negativa, nula y positiva restringida.

\section{Agradecimiento}

Al Consejo Superior de Investigación del Vicerrectorado de Investigación de la UNMSM, por el apoyo financiero otorgado para la ejecución del Proyecto de Estudio de Investigación 2014 con código: 141401111, cuyo resultado es la presente publicación. 


\section{Referencias Bibliográficas}

[1] Cavalcanti, M. M., Cavalcanti, V. D., and Martinez, P. (2008). General decay rate estimates for viscoelastic dissipative systems. Nonlinear Analysis: Theory, Methods and Applications, 68(1): 177-193.

[2] Cavalcanti, M. M., Cavalcanti, V. D., Prates Filho, J. S., and Soriano, J. A. (2001). Existence and uniform decay rates for viscoelastic problems with nonlinear boundary damping. Differential and integral equations, 14(1): 85-116.

[3] Fabrizio, M., and Morro, A. (1992). Mathematical problems in linear viscoelasticity. Society for Industrial and Applied Mathematics.

[4] Ma, J., and Geng, H. (2012). Global nonexistence of arbitrary initial energy solutions of viscoelastic equation with nonlocal boundary damping. Taiwan. J. Math, 16(6): 2019-2033.

[5] Li, F., Zhao, Z., and Chen, Y. (2011). Global existence uniqueness and decay estimates for nonlinear viscoelastic wave equation with boundary dissipation. Nonlinear Analysis: Real World Applications, 12(3): 1759-1773.

[6] Li, M. R., and Tsai, L. Y. (2003). Existence and nonexistence of global solutions of some system of semilinear wave equations. Nonlinear Analysis: Theory, Methods and Applications, 54(8): 1397-1415.

[7] Park, J. Y., and Park, S. H. (2006). Existence and asymptotic stability for viscoelastic problems with nonlocal boundary dissipation. Czechoslovak Mathematical Journal, 56(2): 273-286.

[8] T. Quispe Méndez, T. (2011). Singularidad de soluciones para un sistema de Kirchhoff no lineal viscoelástico con término disipativo. PESQUIMAT, Revista de la F. C. M. de la Universidad Nacional Mayor de San Marcos Vol. XIV(1): 46-57.

[9] Renardy, M., Hrusa, W. J., and Nohel, J. A. (1987). Mathematical problems in viscoelasticity, Pitman Monographs and Surveys in Pure and Applied Mathematics, vol. 35.

[10] Tahamtani, F., and Peyravi, A. (2013). Asymptotic behavior and blow-up of solutions for a nonlinear viscoelastic wave equation with boundary dissipation. Taiwanese J. Math, 17(6): 1921-1943.

[11] Wu, S. T. (2012). General decay and blow-up of solutions for a viscoelastic equation with nonlinear boundary damping-source interactions. Zeitschrift für Angewandte Mathematik und Physik (ZAMP), 63(1): 65-106.

[12] Wu, S. T., and Chen, H. F. (2012). Uniform decay of solutions for a nonlinear viscoelastic wave equation with boundary dissipation. Journal of Function Spaces and Applications, 2012. 